
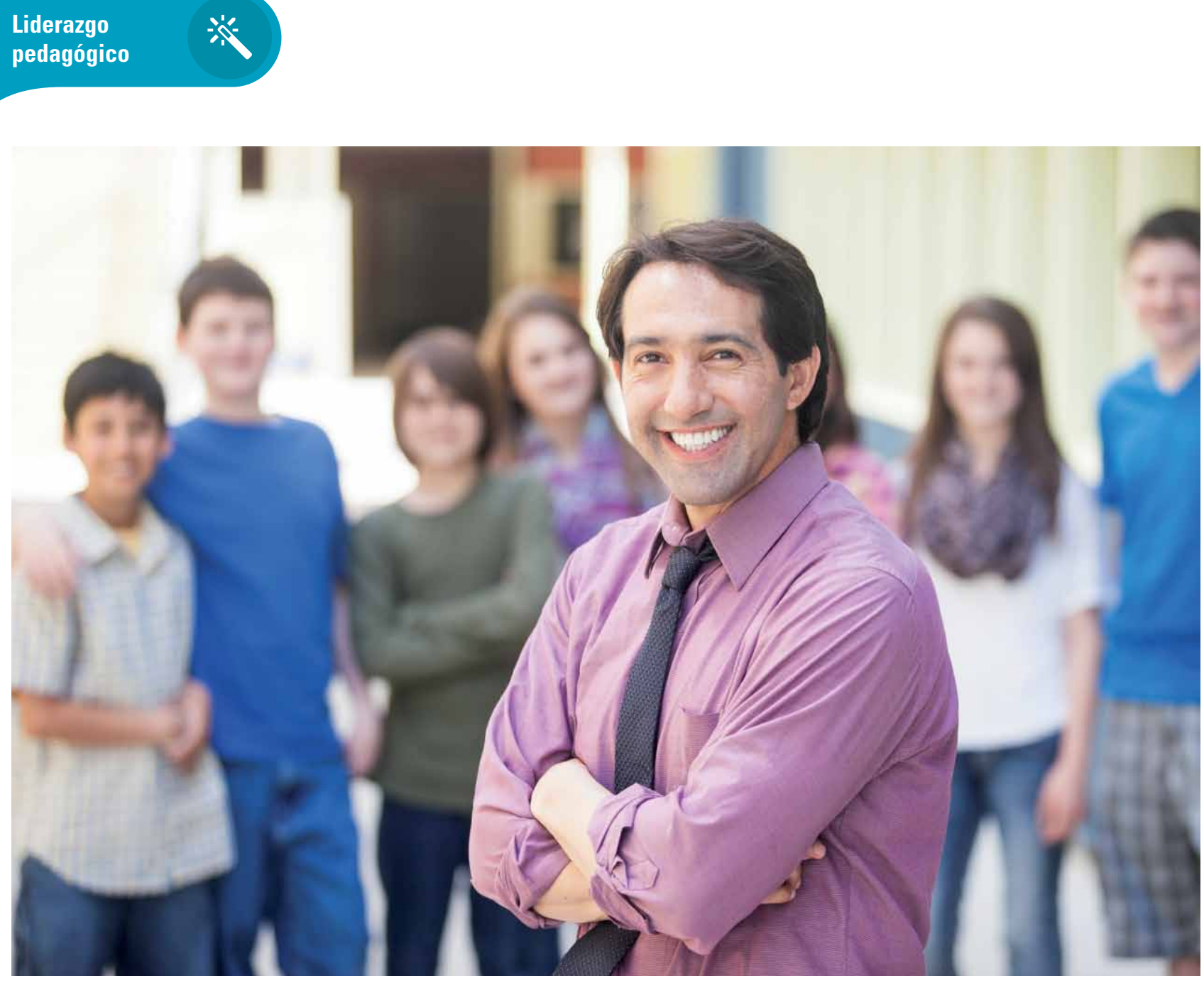

\title{
El liderazgo positivo y compartido
}

Cuando hablamos de liderazgo positivo

y compartido nos referimos sobre todo

a la necesidad de crear en los centros

educativos la cultura del liderazgo, cada

día se hace más apremiante la necesidad

de investigar qué es un buen profesor. Los

estudios que se citan en este artículo, todos

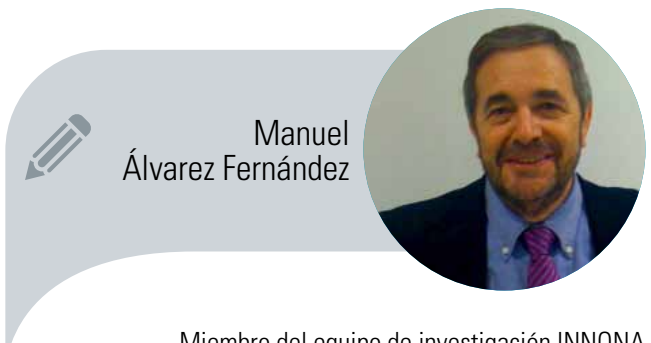

hacen referencia al ejercicio de un liderazgo

Universidad de Deusto manuel.alvarez@deusto.es

afiliativo e integrador como punto de

partida de una nueva cultura que nace del

aula y se extiende a toda la organización. 


\section{Presentación y justificación}

¿Por qué se habla tanto hoy en día de liderazgo? Durante cerca de treinta años, hasta finales de los ochenta y principios de los noventa, se planteaban distintos modelos de dirección de las organizaciones, casi todos, centrados en el sujeto que desempeñaba el rol de dirigir. En la década de los noventa, Burns y Bass comienzan a definir un tipo de liderazgo centrado en las personas y su relación con ellas. Estos autores desplazan el centro de la investigación desde la dirección del sujeto que ejerce el liderazgo a la interacción de éste con sus colaboradores. Más tarde, Goleman aportó el enfoque de la influencia de la inteligencia emocional al desarrollo del liderazgo compartido.

Como se podrá suponer, este proceso de investigación surge porque es precisamente por esta época cuando comienzan a cambiar drásticamente las condiciones del mercado y de la producción juntamente con el contexto del trabajo gracias a la aparición de las TIC y de la tecnología informática. A los factores clásicos del capital y de la producción en la organización de corte capitalista surge lo que Peter Drucker (1993) llama la "sociedad del conocimiento" en la que la innovación, la comunicación y el conocimiento generan un nuevo tipo de trabajador cuyo perfil profesional va unido a conceptos como autonomía, autodesarrollo profesional, dominio de los medios de producción, motivación por el logro y desempeño.

Todo esto supone una nueva organización de los procesos de producción, de la relación con las personas y de la generación del conocimiento que exigen un nuevo liderazgo capaz de transmitir confianza y activar y movilizar las energías creativas e innovadoras de las personas.

\section{El nuevo liderazgo emergente}

Ya en la década del 2000 las nuevas aportaciones a la investigación sobre el liderazgo como un factor clave en la obtención de resultados se centran en conceptos tales como empatía, competencias sociales, motivación, resonancia, compasión, coaching, resiliencia, conceptos que hacen referencia a la implicación de los profesionales colaboradores en proyectos atractivos y sugerentes de las organizaciones.

Goleman, es el primer autor que además de hacer una aportación decisiva con el modelo de inteligencia emocional, define por primera vez el liderazgo positivo como "la capacidad de algunos líderes de sintonizar con los sentimientos de las personas y encauzarlos en una dirección emocionalmente po-

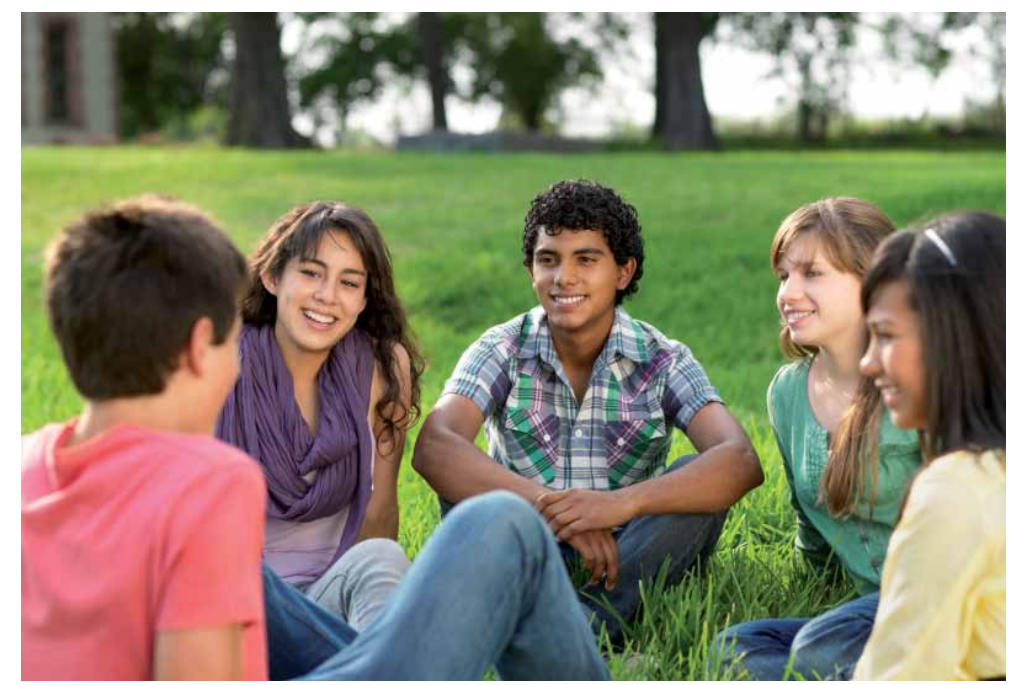

sitiva". También contempla la posibilidad del liderazgo negativo, disonante, como la falta de armonía y conexión con las emociones de sus subordinados. La resonancia es el modo en que el líder ejerce los distintos estilos de liderazgo: aquellos que alientan la resonancia (visionario, coaching, afiliativo y democrático) y los que sólo son útiles en situaciones concretas porque alientan la disonancia como timonel y autoritario.

El líder más eficaz sería aquel que se sirve de los seis estilos de liderazgo en función del contexto, de la situación y del momento del desarrollo de la organización.

Antes, Russell Swann, (2000) introduce un nuevo concepto que llama "liderazgo compasivo de género" como resultado de su investigación en las organizaciones de servicios lideradas por la mujer. Los trabajos inspirados en este enfoque demuestran que el concepto de liderazgo ha sido construido sobre los parámetros masculinos de agresividad, dominio, independencia y control emocional marginando la voz, los sentimientos, las ideas y planteamientos de la mujer. Éstas tienden a expresar el poder de forma más cooperativa, dando más importancia a las redes de comunicación informales, propiciando la cohesión y los vínculos entre los miembros, alentando a compartir y a trabajar en equipo para conseguir objetivos previamente consensuados.

Swann, entiende por compasión "un sentimiento de dolor o pena por el sufrimiento o desgracia del otro". Más adelante matiza, diferenciando pena de compasión y vuelve a definir el concepto de compasión como solidaridad compartida con el sufrimiento 0 gozo de los demás. Este nuevo planteamiento significa según sus palabras "celebración (disfrute con las alegrías o logros del otro y sufrimiento con sus amarguras o fracasos)".

La compasión no es un mero proceso mental; sino que implica el sentimiento y la acción que tiene por fin aliviar la desgracia del otro. 


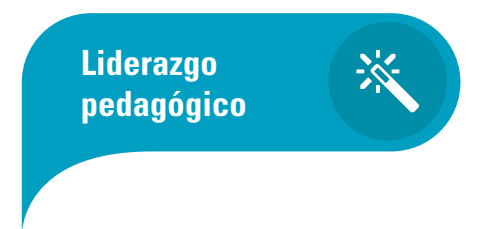

Conocedor, de la realidad profunda de la escuela en los suburbios de Victoria, Australia, se plantea la necesidad de este nuevo modelo de dirección a partir de los datos que arroja la situación social y econó-

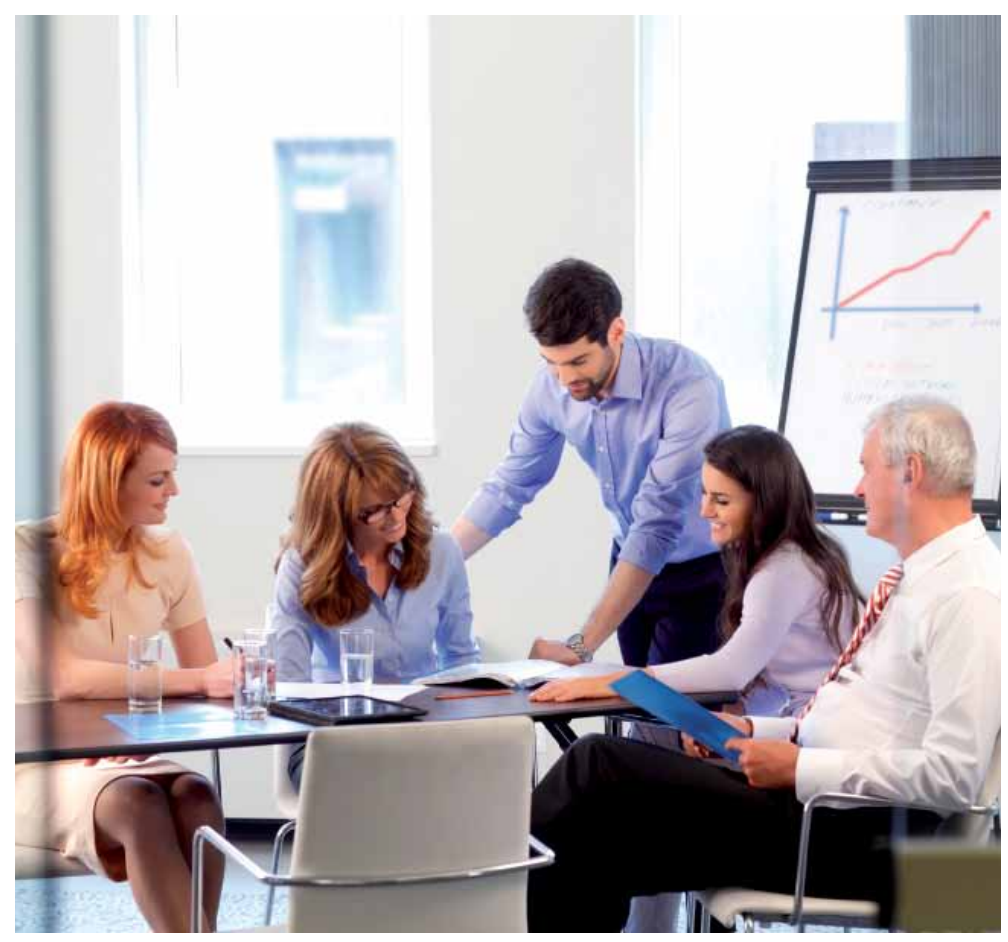

mica del alumnado de este país, no tan lejano a las costumbres y cultura de Occidente. El aumento de la enseñanza obligatoria hasta los dieciséis o dieciocho años, la familia desestructurada, el desempleo, la falta de interés de los alumnos y las dificultades de aprendizaje debidas a la desintegración social y al sentido de no pertenencia y de alineación de su propio hogar, le llevan a un replanteamiento radical de la misión y visión de futuro de la escuela.

La pregunta clave que abre la perspectiva de una investigación sobre el nuevo perfil de dirección que necesita la nueva escuela es: " ¿puede un director ser compasivo, gestionar con éxito y además sobrevivir?"

Dice Leithwood (2009) que la investigación sobre el liderazgo positivo y distribuido se encuentra en un estado embrionario pero el interés y el número de investigaciones es muy alto. Todo parte del descubrimiento de que el ejercicio de un liderazgo compartido constituye un factor clave de influencia indirecta en los resultados y aprendizaje de los alumnos. Se pretende diseñar un modelo de liderazgo de organización de tal forma que tanto el equipo directivo como el profesorado ejerzan el mismo modelo de influencia sobre las creencias, valores y acciones de los otros. Tanto los equipos

\section{El líder más eficaz sería aquel que se sirve de los seis estilos de liderazgo en función del contexto, de la situación y del momento del desarrollo de la organización}

directivos como el profesorado participan de los mismos objetivos a conseguir mediante su influencia, para que los alumnos se impliquen en el proceso de aprendizaje. Los directores pueden influir directamente, dice Leithwood (2009: páginas 21 y 22) creando un buen clima de trabajo, facilitando el trabajo a los profesores, seleccionando y reemplazando de manera más rigurosa al personal del centro, monitoreando la actividades escolares, proporcionando recursos funcionales de tiempo y conocimiento al profesorado.

Indirectamente los directivos también pueden influir sobre otros factores relevantes del aprendizaje de los alumnos como la participación de los padres y el seguimiento de las tareas escolares de sus hijos, el desarrollo de esquemas eficaces de agrupamiento flexible de los alumnos y otras disposiciones organizativas, apoyando siempre a los profesores y creando ámbitos de resiliencia que les ayuden a superar los momentos difíciles de la profesión.

El liderazgo de los profesores puede ser muy variado y va desde el liderazgo de influencia directa en el proceso de aprendizaje de los alumnos en el aula, hasta el liderazgo intermedio de coordinación de la tarea educativa en los equipos docentes, dirección de departamentos o equipos de orientación.

Tanto el liderazgo de los directores como el de los profesores en un modelo de liderazgo distribuido implica un ejercicio de influencia sobre creencias, valores y hábitos de socialización.

Como síntesis, "el liderazgo distribuido supone un conjunto de prácticas para fijar el rumbo y ejercer influencia sobre personas distribuidas en todos los niveles de la organización escolar más que un 
conjunto de atributos y características personales en la cúspide de la organización".

\section{Modelo de liderazgo positivo y compartido en el contexto escolar}

Este marco conceptual nos permite precisar de forma más rigurosa y consistente lo que entendemos por liderazgo positivo y compartido en el contexto actual de la escuela en nuestro país.

El propósito de este modelo de liderazgo emergente es crear una cultura colectiva en que se valoren y gestionen las emociones positivas de un grupo como la alegría, la confianza, el amor, la inclusión, el reconocimiento, la aceptación, la satisfacción profesional, reduciendo y controlando las negativas como el miedo, la ira, la ansiedad, la exclusión, la tristeza o el sentido de fracaso personal. Todos los estudios sobre las organizaciones que han superado situaciones de máximo estrés y desestructuración interna coinciden en afirmar que promover emociones positivas mejora la motivación y el bienestar de los profesionales lo que se traduce en un aumento de creatividad, compromiso y capacidad de adaptación a situaciones nuevas. Los directivos de éxito que lideran estos procesos de resiliencia se han centrado en "lo que funciona", en "los factores críticos de éxito" de la nueva organización y en "Io positivo" de cada colaborador como punto de partida de la superación.

Los factores que hoy en día se están estudiando (Leithwood, Goleman, Boyatzis, Cyrulnick, La Puerta y Shahar) como descriptores del modelo de liderazgo positivo son los siguientes:

$\boldsymbol{\lambda}$ La implicación. Probablemente la capacidad que mejor definen el liderazgo profesional de los directivos se refiera a la capacidad de implicar a su gente en un nuevo proyecto y gestionar las resistencias frente a los cambios necesarios. Implicar, incluir, integrar suponen habilidades que hacen referencia a factores de liderazgo transformacional y emocional como la consideración individual, la motivación, el reconocimiento, la empatía, en resumidas cuentas a la competencia de relación personal y social.

Se puede implicar e influir en la gente de muchas maneras:

a. Mediante la aplicación estricta de la normativa y la sanción por su incumplimiento, propias de la dirección administrativa que fomentan el miedo y la sumisión.

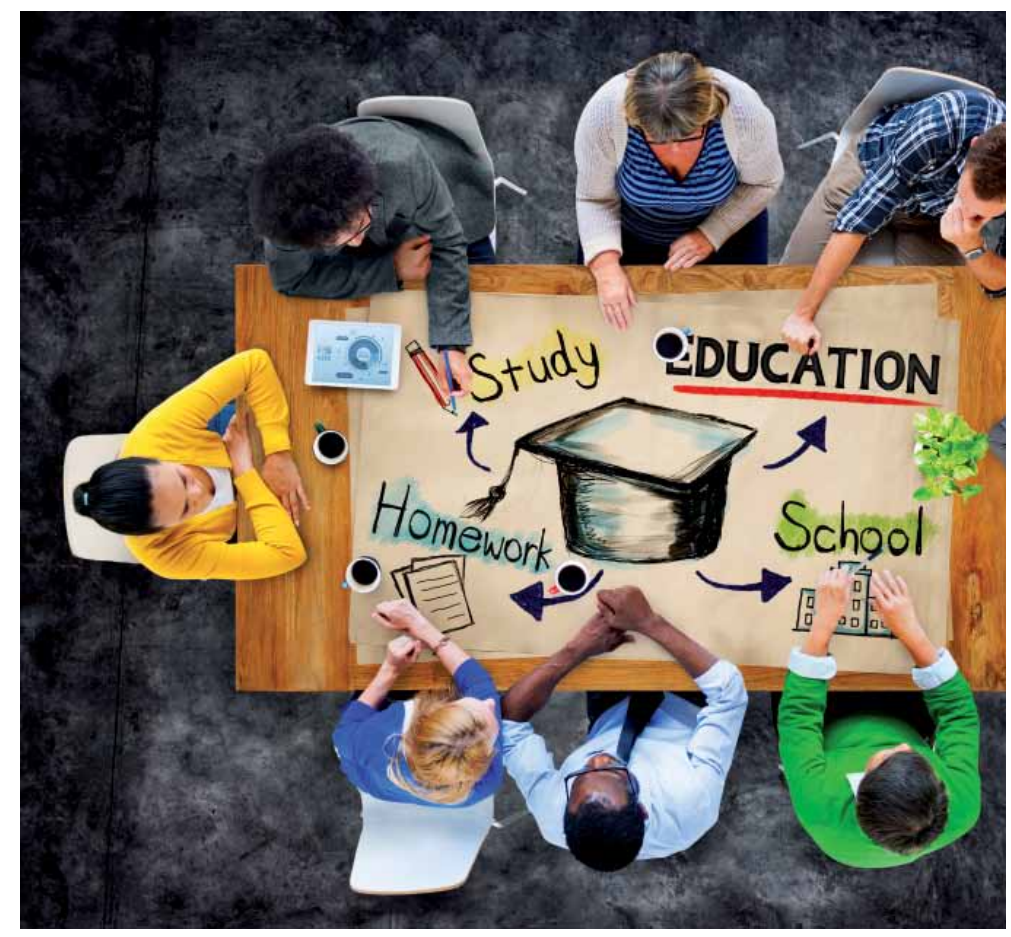

b. A través de la negociación de los estándares y objetivos a alcanzar mediante incentivos que motiven un mayor rendimiento. Este modelo es propio del liderazgo transaccional y fomenta la competitividad.

c. Finalmente, a través de la generación del compromiso personal y colectivo de sus colaboradores, lo que fomenta la motivación por el logro y a la colaboración para obtener mejores resultados que satisfagan y beneficien al individuo y a la organización. Éste es el proceso de inclusión que prima en el liderazgo positivo.

$\boldsymbol{\lambda}$ La innovación. Siempre se ha considerado al liderazgo como facilitador del cambio y estimulador de la innovación. Puertas y Shahar (2012) sostienen que la raíz del cambio está en visualizar los comportamientos que necesita la organización para conseguir y mantener los cambios. Los procesos tradicionales de cambio se centran únicamente en aspectos tangibles como procesos, estrategias y herramientas. Se olvidan de que el cambio supone nuevos comportamientos de las personas y que el verdadero cambio está en conseguir que la gente trabaje de otra manera. Las únicas personas capaces de generar comportamientos de alto rendimiento son los líderes 


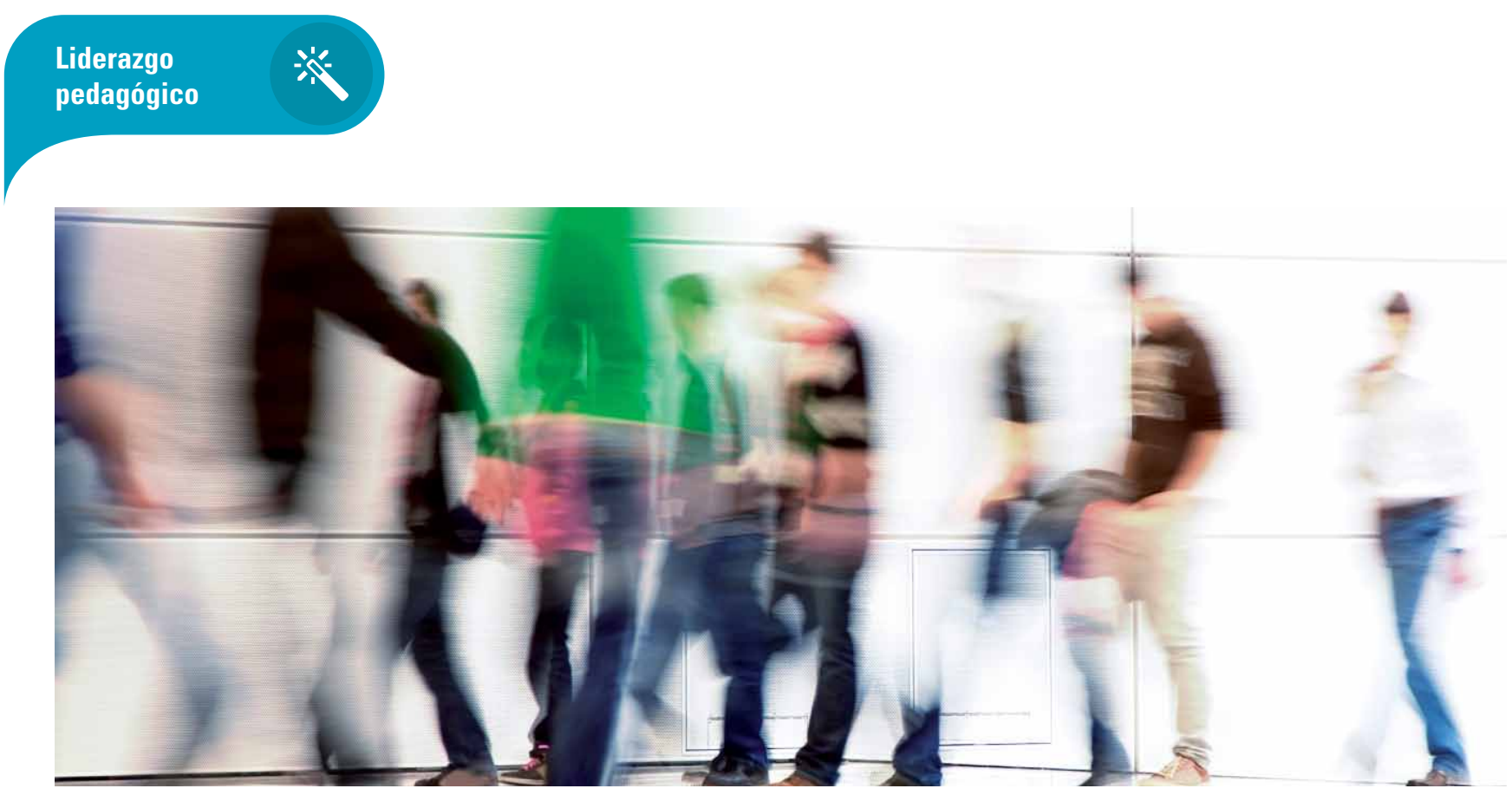

que ejercen un liderazgo positivo como guías, facilitadores, gestores de las resistencias y de los elementos tóxicos que dificultan los procesos y la institucionalización de nuevos comportamientos. Los procesos de cambio como la mayor parte de los procesos que hacen viables las organizaciones radican en las personas y en su capacidad de adaptarse a las nuevas situaciones y tecnologías.

7 La influencia. Para conseguir que las personas se impliquen en procesos de cambio que exigen esfuerzos y energía por encima de sus obligaciones, es necesaria la presencia de un liderazgo con la suficiente credibilidad como para hacer necesario el compromiso que exige la organización. En el fondo, influir en otros para que hagan lo que alguien sugiere son necesarias tres cualidades:

1. Autoridad, sobreviene porque la persona ha demostrado sus logros y su competencia profesional a través de la experiencia.

2. Confianza que inspira en sus seguidores, porque siempre ha dado ejemplo de honestidad, transparencia y compromiso con la palabra dada.

3. Seguridad que surge por la trayectoria de éxito y resultados que garantizan la protección en el puesto de trabajo y un futuro mejor de la organización

4. Comunicación convincente como la capacidad de convencimiento que implica explicación clara y razonable de los nuevos comportamientos exigibles para los cambios que precisa la institución y el control de los riesgos que se corren.

7 El coaching. Para proporcionar seguridad a la gente y conseguir que se suban al tren de los cambios es imprescindible también proporcionar formación y facilitar el acceso a nuevos conocimientos que desarrollen habilidades desconocidas. Esta labor, que no siempre puede llevar a cabo el equipo líder, sí precisa sin embargo la presencia de un programa consistente de formación y asesoramiento, así como la presencia constante del liderazgo en los entrenamientos, demostrando que se cree en los cambios. No basta solo con facilitar recursos de tiempo y apoyo intencionado, la función de coaching significa también compromiso y presencia.

7 Resiliencia. Según Cyrulnick (2012) resiliencia es la característica de ciertos materiales para recomponerse inmediatamente después de un golpe violento. El entorno profesional de ciertas organizaciones de servicio se ha vuelto traumático como consecuencias de la crisis económica, la presión del cambio, los altos niveles de incertidumbre y ambigüedad en puestos de trabajo inseguros, así como el alto nivel de exigencia y rendimiento que plantean las empresas con objetivos cambiantes e imprevistos.

Todo esto lleva a ciertos individuos al borde de la desestructuración personal con crisis de ansiedad, angustia y deseo de huida en el más absoluto silencio.

En este contexto, más frecuente del que se percibe a simple vista, se hace necesaria la presencia de un liderazgo capaz de rescatar al colaborador 0 al equipo de la caída en el precipicio. No son necesarias la mayor parte de las veces las habilidades y competencias profesionales del terapeuta o del psiquiatra, sencillamente se necesita un liderazgo capaz de crear un clima positivo de confianza, optimismo y relación personal que algunos Ilaman amor y otros amabilidad.

\section{El liderazgo positivo del buen profesor.}

El liderazgo positivo compartido se distribuye en tres ámbitos cuya interacción y acción con- 
gruente es fundamental para que sea operativo y eficaz: el ámbito de gobierno y dirección, el ámbito intermedio de coordinación profesional y el ámbito clave del aula. Este último, tan importante en el liderazgo docente, está todavía por desarrollar en la investigación. Ahora empiezan a editarse estudios sobre el buen profesor: Bain, (2007), Day y Gu, (2012), Marcelo y Vaillant, (2013), que se acercan al modelo de liderazgo que preconizamos.

¿Por qué es necesario investigar sobre el buen profesor? Las organizaciones de servicio como sanidad, justicia, educación, (Welfare) bienestar social, están orientadas a proporcionar servicio a las personas. Estas organizaciones se diferencian de las empresariales y comerciales por un conjunto de factores que las hace peculiares e importantes como:

7 La intangibilidad. El producto ni se puede tocar ni almacenar porque es un bien como la salud, la libertad, la educación, el aprendizaje o el bienestar, bienes que solo se perciben en sus efectos.

入 La interacción. El bien o servicio sólo puede producirse en la relación personal e intransferible entre el productor y el cliente

$\boldsymbol{7}$ La calidad. Ésta aparece fuertemente condicionada por el nivel de satisfacción tanto del productor en su trabajo como del usuario por el trato recibido.

Es fácil concluir la importancia que adquiere la presencia de un liderazgo positivo a la hora de implicar y motivar a un profesorado del que depende fundamentalmente la calidad del servicio que ofrece la escuela. El nivel de satisfacción tanto de la familia como del alumno se explica por la competencia del profesor, por su capacidad "consideración individual, por su "compasión" y empatía, por su habilidad para implicar a los alumnos en el proceso educativo. En resumidas cuentas por el tipo de interacción que se establece entre el profesor y el niño. Para facilitar el desarrollo de estas capacidades docentes es fundamental que el director conozca profesionalmente qué es ser un buen profesor, que esté al día de las competencias que debe desarrollar un buen docente en el contexto social complejo y variable que nos ha tocado vivir. Es fundamental, en resumidas cuentas conocer las investigaciones sobre lo que significa ser un buen docente y lo que la sociedad y el alumno esperan de un buen profesor •

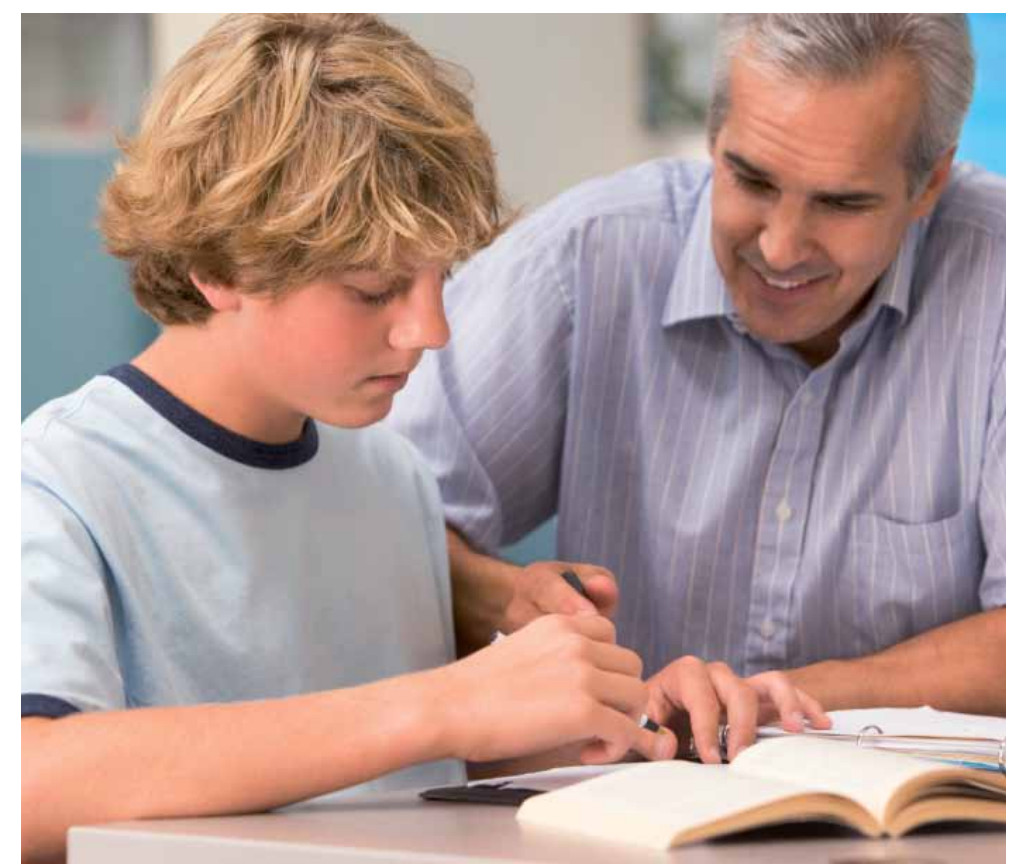

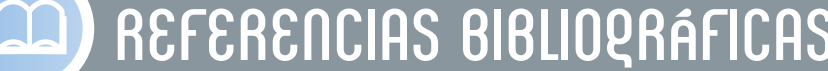

Álvarez, M. (2002). "El liderazgo compasivo de Russell Swann". Capítulo 9: El ejercicio de liderazgo en la escuela. En Dirección escolar ante los retos del siglo XXI. Madrid. Síntesis , pp. 245-246.

Bain, Ken. (2007). Lo que hacen los mejores profesores universitarios. Valencia: Universidad de Valencia

BASs B.M. y Avouı, B. (1993). El liderazgo transformacional y la cultura organizacional. Trimestral administración pública. p. 112 a 121. Chile

CYrulnick, B. (2013). Los Patitos feos. Barcelona: Gedisa

Day, CH. y Gu, 0. (2012). Profesores: vidas nuevas verdades. Narcea. Madrid

Drucker P.F. (1993). La sociedad postcapitalista. Barcelona: Apóstrofe

Drummond, E. (1995). Qué es hoy la Calidad Total. Bilbao: Deusto

Goleman, D. y BoyAtzIs, R. (2002). El líder resonante crea más, el poder de la inteligencia emocional. Barcelona: Taurus, Ariel

LeiHwood, K. (2009). Como liderar nuestras escuelas, aportes desde la investigación.

Marcelo, C y Vaillant , D. (2013). Desarrollo profesional docente Cómo aprender a enseñar. Madrid: Narcea

Pascual, R. Villa, A y Auzmendi, E. (1993). El liderazgo transformacional de los centros docentes. Bilbao: Mensajero

Puertas, F. y ShaHAR, T.B. (2012). Liderazgo positivo para el desarrollo de la organización y una cultura de alto rendimiento. Bilbao: Harvard Deusto Business

\section{HEMOS HABLADO DE}

\section{Liderazgo pedagógico, dirección escolar, calidad educativa, liderazgo transformacional.}

Este artículo fue solicitado por PADRES Y MAESTROS en octubre de 2014, revisado y aceptado en enero de 2015 para su publicación. 